\title{
Electron inventory of the iron-sulfur scaffold complex HypCD essential in [NiFe]-hydrogenase cofactor assembly
}

\author{
(Dven T. Stripp ${ }^{1}$, Jonathan Oltmanns ${ }^{2}$, Christina S. Müller ${ }^{2}$, David Ehrenberg ${ }^{1}$, Ramona Schlesinger ${ }^{3}$, \\ Joachim Heberle ${ }^{1}$, Lorenz Adrian ${ }^{4,5}$, Volker Schünemann ${ }^{2}$, Antonio J. Pierik ${ }^{6}$ and ${ }^{\circ}$ Basem Soboh $^{3}$
}

${ }^{1}$ Experimental Molecular Biophysics, Department of Physics, Freie Universität Berlin, Arnimallee 14, 14195 Berlin, Germany; ${ }^{2}$ Biophysics and Medical Physics, Department of Physics, Technische Universität Kaiserslautern, Erwin-Schrödinger-Straße 56, 67663 Kaiserslautern, Germany; ${ }^{3}$ Genetic Biophysics, Department of Physics, Freie Universität Berlin, Arnimallee 14, 14195 Berlin, Germany; ${ }^{4}$ Department of Environmental Biotechnology, Helmholtz Centre for Environmental Research - UFZ, Permoserstraße 15, 04318 Leipzig, Germany; ${ }^{5}$ Chair of Geobiotechnology, Technische Universität Berlin, Ackerstraße 76, 13355 Berlin, Germany; ${ }^{6}$ Biochemistry, Department of Chemistry, Technische Universität Kaiserslautern, Erwin-Schrödinger-Straße 54, 67663 Kaiserslautern, Germany

Correspondence: Basem Soboh (basem.soboh@fu-berlin.de)

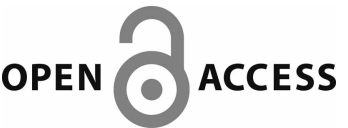

Received: 30 March 2021 Revised: 17 August 2021 Accepted: 19 August 2021

Accepted Manuscript online: 19 August 2021

Version of Record published: 7 September 2021
The [4Fe-4S] cluster containing scaffold complex HypCD is the central construction site for the assembly of the $[\mathrm{Fe}](\mathrm{CN})_{2} \mathrm{CO}$ cofactor precursor of [NiFe]-hydrogenase. While the importance of the HypCD complex is well established, not much is known about the mechanism by which the $\mathrm{CN}^{-}$and $\mathrm{CO}$ ligands are transferred and attached to the iron ion. We report an efficient expression and purification system producing the HypCD complex from $E$. coli with complete metal content. This enabled in-depth spectroscopic characterizations. The results obtained by EPR and Mössbauer spectroscopy demonstrate that the $[\mathrm{Fe}](\mathrm{CN})_{2} \mathrm{CO}$ cofactor and the $[4 \mathrm{Fe}-4 \mathrm{~S}]$ cluster of the HypCD complex are redox active. The data indicate a potential-dependent interconversion of the $[\mathrm{Fe}]^{2+/ 3+}$ and $[4 \mathrm{Fe}-4 \mathrm{~S}]^{2+/+}$ couple, respectively. Moreover, ATR FTIR spectroscopy reveals potentialdependent disulfide formation, which hints at an electron confurcation step between the metal centers. MicroScale thermophoresis indicates preferable binding between the HypCD complex and its in vivo interaction partner HypE under reducing conditions. Together, these results provide comprehensive evidence for an electron inventory fit to drive multi-electron redox reactions required for the assembly of the $\mathrm{CN}^{-}$and $\mathrm{CO}$ ligands on the scaffold complex HypCD.

\section{Introduction}

Gas-processing metalloenzymes facilitate complex and essential metabolic processes such as photosynthesis, respiration, and $\mathrm{N}_{2}$ fixation [1]. Hydrogenases catalyze the reversible conversion of $\mathrm{H}_{2}$ into protons and electrons. Catalysis occurs at bimetallic active sites that contain either nickel and iron or only iron [2]. The nature of the metallic cofactor forms the basis of the distinction of hydrogenases into three classes: $[\mathrm{FeFe}]-, \quad[\mathrm{NiFe}]-$, and iron sulfur cluster-free hydrogenases [3,4]. [NiFe]-hydrogenase and $[\mathrm{FeFe}]$-hydrogenase are unrelated at the amino acid sequence level but their cofactors share the unique feature of having diatomic ligands like carbonyl $(\mathrm{CO})$ and cyanide $\left(\mathrm{CN}^{-}\right)$, each associated with the iron ions. To obtain a clear understanding of these specific structural features, studies of the biosynthesis, structure, and mechanism of $\mathrm{H}_{2}$ activation at the molecular level are important $[5,6]$. There is also interest from applied research because it is hoped to learn from hydrogenase how a robust catalyst for reversible $\mathrm{H}_{2}$ activation can be developed [6]. The focus of this work is on the biosynthesis of [NiFe]-hydrogenase.

Standard [NiFe]-hydrogenase consists of a large subunit and a small subunit. The large subunit contains the catalytic $[\mathrm{NiFe}](\mathrm{CN})_{2} \mathrm{CO}$ cofactor whereas the small subunit features three iron-sulfur clusters involved in electron transfer between the active site and redox partners like ferredoxin or 
flavodoxin [2,4]. Additional subunits may be present to transfer the electrons to pyridine nucleotides or to anchor the multimeric enzyme to the membrane [7-9]. Despite these structural differences, the catalytic cofactor is strictly conserved among all [NiFe]-hydrogenases. The nickel ion is coordinated by four cysteinyl thiolates in the active site, while two of the thiolates additionally bind an iron ion. The iron site carries one $\mathrm{CO}$ and two $\mathrm{CN}^{-}$ligands $[10,11]$. The ligands are proposed to maintain the low-spin $\mathrm{Fe}^{2+}$ state important for $\mathrm{H}_{2}$ activation. If these ligands are not attached to the iron ion, no active hydrogenase enzyme can be synthesized [12].

A complex machinery is involved in the synthesis and insertion of the $[\mathrm{NiFe}](\mathrm{CN})_{2} \mathrm{CO}$ cofactor into the apo-enzyme [12,13]. Figure 1 presents our current working model for hydrogenase maturation and suggests the following sequence of events: (i) Synthesis of $\mathrm{CO}$ and $\mathrm{CN}^{-}$, ligation to iron, and incorporation of the fully coordinated iron into the large subunit pro-protein by the coordinated activity of HypCDEF (see below) [13-15]; (ii) Nickel insertion by concerted activity of HypA, HypB, and SlyD $[14,16]$. (iii) Cleavage of the $C$-terminal extension of the pro-protein by a specific protease that recognizes the completed metal cluster, resulting in a conformational change that locks the active site in place [13]. (iv) Finally, dimerization of the large subunit with the



Figure 1. Working model for maturation of the large subunit into functional [NiFe]-hydrogenase.

(A) (i) Incorporation of the $\mathrm{Fe}(\mathrm{CN})_{2} \mathrm{CO}$ moiety into the hydrogenase large subunit (pro-protein) by the coordinated activity of HypCDEF (dashed arrow). (ii) Nickel insertion. (iii) Proteolytic cleavage of the C-terminal peptide, associated with conformational changes of the protein. (iv) Dimerization of large and small subunit to form functional [NiFe]-hydrogenase. (B) The bottom half, biosynthesis of the $[\mathrm{Fe}](\mathrm{CN})_{2} \mathrm{CO}$ moiety. (1) The HypCD complex is formed upon contact with the $[\mathrm{Fe}] \mathrm{CO}_{2}$ carrying HypC dimer. (2) The $\mathrm{CO}_{2}$ ligand may undergo an ATP-dependent reduction to $\mathrm{CO}$ catalyzed by HypCD complex. (3) The $\mathrm{CN}^{-}$ligands are generated by maturation proteins HypE and HypF. (4) Transfer of the $\mathrm{CN}^{-}$ligands to the CO-modified iron ion and formation of the $[\mathrm{Fe}](\mathrm{CN})_{2} \mathrm{CO}$ precursor on the HypCD complex. 
small subunit to yield active enzyme [12,17-19]. To date, it is not clear where the iron ion in the active site originates from, how the diatomic ligands are attached to the cofactor precursor, and how the CO ligand is synthesized. In the following, we will focus on the biosynthesis of $[\mathrm{Fe}](\mathrm{CN})_{2} \mathrm{CO}$ cofactor precursor. Our working hypothesis for the assembly of $[\mathrm{Fe}](\mathrm{CN})_{2} \mathrm{CO}$ moiety suggests the following steps (Figure $\left.1 \mathrm{~B}\right)$. (1) The HypC dimer delivers both iron and $\mathrm{CO}_{2}$, the later which represents a putative precursor of the $\mathrm{CO}$ ligand [20]. Subsequently, the HypCD complex is formed upon contact with HypD while a HypC monomer is released [20]. (2) The HypCD complex may catalyze an ATP-dependent reduction in $\mathrm{CO}_{2}$ to $\mathrm{CO}$ at the expense of 1 eq. of $\mathrm{H}_{2} \mathrm{O}$ [21]. The source of electrons required for the reduction in $\mathrm{CO}_{2}$ to $\mathrm{CO}$ is unknown. (3) The $\mathrm{CN}^{-}$ligands are generated by the maturation proteins HypE and HypF [22]. (4) Transfer of the $\mathrm{CN}^{-}$ligands to the CO-modified iron ion and formation of the $[\mathrm{Fe}](\mathrm{CN})_{2} \mathrm{CO}$ precursor on the HypCD complex [23,24]. Insertion of the cofactor precursor into the large subunit takes place by the coordinated activity of four maturation proteins, HypCDEF $[12,19]$. This working hypothesis is supported by the isolation and characterization of complex intermediates of Hyp-maturation proteins, e.g. HypC $[\mathrm{Fe}] \mathrm{CO}_{2}$, HypCD $[\mathrm{Fe}] \mathrm{CO}_{2}$, HypCD $[\mathrm{Fe}] \mathrm{CO}$, and $\mathrm{HypCD}[\mathrm{Fe}](\mathrm{CN})_{2} \mathrm{CO}$. The binding of $\mathrm{CN}^{-}, \mathrm{CO}$, and $\mathrm{CO}_{2}$ ligands in these complexes correlates with the presence of a supernumerary iron ion along with the [4Fe-4S] cluster in HypD [24]. Replacement of conserved amino acids in HypC and HypD abolished iron binding and resulted in the loss of the ligands' absorption bands [20,25]. These findings indicate functional coupling between $\mathrm{Fe}, \mathrm{CO}, \mathrm{CN}^{-}$, and $\mathrm{CO}_{2}$.

The HypCD complex is the central synthesis platform, where $\mathrm{CO}$ and $\mathrm{CN}^{-}$ligands are synthesized and attached to the iron ion $[24,26,27]$. Cyanation would require the input of two electrons each, if the transfer takes place by an electrophilic substitution mechanism [26]. Additionally, two electrons for $\mathrm{CO}_{2}$ reduction will be required if $\mathrm{CO}_{2}$ is the metabolic precursor of $\mathrm{CO}$ [28]. It is likely that the HypD protein is involved in this electron transfer reaction, since HypD is the only maturation protein that carries an iron-sulfur cluster and therefore it is the only Hyp protein potentially capable of redox chemistry. However, the redox activity of the [4Fe-4S] cluster has not been demonstrated yet.

In the present work, we developed an efficient expression and purification system to produce the HypCD complex with complete cluster and cofactor content. This facilitated a comprehensive spectroscopic characterization, combining UV/Vis, resonance Raman, ATR FTIR, EPR, and Mössbauer spectroscopy. Our study indicates that the [4Fe-4S] cluster and the mononuclear iron site of the HypCD complex are redox active. Moreover, we investigate the binding affinities of HypCD to its in vivo interaction partner HypE and the HypEF complex using MicroScale thermophoresis (MST).

\section{Materials and methods}

\section{Bacterial strains, plasmids, and growth conditions}

Plasmid pT-hypDEFC [26] was used to generate pT-hypDEFC ${ }^{\text {StrepXT }}$ for overproduction of the recombinant HypCD complex that has two strep-tag sequences (Twin-Strep-tag) with an internal linker region fused to HypC as C-terminal tag. The HypCD complex was overexpressed in E. coli strain MC4100 (DE3) transformed with the pT-hypDEFC ${ }^{\text {StrepXT }}$ plasmid. Large scale protein purification was performed with 20 liters of anaerobic TGYEP full medium ( $1 \%$ tryptone, $0.5 \%$ yeast extract) at $37^{\circ} \mathrm{C}$ with a typical yield of $3-4 \mathrm{~g}$ of cells/liter. The growth medium contained $100 \mu \mathrm{g} / \mathrm{ml}$ ampicillin and was supplemented with $0.8 \%(\mathrm{~m} / \mathrm{v})$ glucose and $10 \mathrm{mM}$ sodium formate. To induce gene expression, isopropyl- $\beta$-D-thiogalactopyranosid (IPTG) was added to a final concentration of $50 \mu \mathrm{M}$ at $\mathrm{OD}_{600 \mathrm{~nm}} \sim 0.3$. After induction, the culture was incubated at $25^{\circ} \mathrm{C}$ for a further 5-8 $\mathrm{h}$ or after the culture reached an optical density between $0.8-1.2$ (exponential growth, see Supplementary Figure S1). Cells were harvested by centrifugation at $15000 \mathrm{~g}$ for $20 \mathrm{~min}$ at $4^{\circ} \mathrm{C}$. Cell pellets were used either immediately or stored at $-80^{\circ} \mathrm{C}$.

To produce the ${ }^{57} \mathrm{Fe}$-labeled HypCD complex for Mössbauer spectroscopy, the bacteria were grown anaerobically either in M9 minimal medium (M9 salt, $0.4 \%$ glucose (m/v), $0.3 \mathrm{mM} \mathrm{MgSO} 4,0.3 \mathrm{mM} \mathrm{CaCl}, 1 \mu \mathrm{g} / \mathrm{l}$ biotin, $1 \mu \mathrm{g} / \mathrm{l}$ thiamine and trace elements) or modified minimal medium (M9*) or modified full medium $\left(\right.$ TGYEP $\left.^{\star}\right)$. The later contained only $0.2 \%$ yeast extract to reduce the natural occurring ${ }^{56} \mathrm{Fe}$ content. At the time of gene induction, ${ }^{57} \mathrm{Fe}$ was added to TGYEP ${ }^{\star}$ medium to a final concentration of $20 \mu \mathrm{M}$ or $100 \mu \mathrm{M}$ (the growth curve in Supplementary Figure S1 shows TGYEP* $+20 \mu \mathrm{M}{ }^{57} \mathrm{Fe}$ ). The M9* medium contained the following compounds: $34 \mathrm{mM} \mathrm{Na}_{2} \mathrm{HPO}_{4}, 22 \mathrm{mM} \mathrm{KH}_{2} \mathrm{PO}_{4}, 8.5 \mathrm{mM} \mathrm{NaCl}, 9.35 \mathrm{mM} \mathrm{NH} \mathrm{Nl}_{4} \mathrm{Cl}, 0.4 \%$ (m/v) glucose, $0.3 \mathrm{mM} \mathrm{MgSO}_{4}, 0.3 \mathrm{mM} \mathrm{CaCl}_{2}, 1 \mu \mathrm{g} / \mathrm{l}$ biotin, $1 \mu \mathrm{g} / \mathrm{l}$ thiamine, $0.2 \%$ peptone, and $10 \mathrm{mM} \mathrm{Na}$ formate. The following trace elements were added: $134 \mu \mathrm{M}$ EDTA, $6 \mu \mathrm{M} \mathrm{ZnCl}_{2}, 0.76 \mu \mathrm{M} \mathrm{CuSO}_{4}, 0.42 \mu \mathrm{M} \mathrm{CoCl} 2,1.6 \mu \mathrm{M}$ 
$\mathrm{H}_{3} \mathrm{BO}_{3}$, and $0.08 \mu \mathrm{M} \mathrm{MnCl}$. At the time of gene induction, $20 \mu \mathrm{M}{ }^{57} \mathrm{Fe}$ was added to the medium. Cells were harvested in the exponential growth phase and washed twice with $100 \mathrm{mM}$ Tris- $\mathrm{HCl}$ buffer $\mathrm{pH} 8$ containing $150 \mathrm{mM} \mathrm{NaCl}$ and $1 \mathrm{mM}$ EDTA.

\section{Preparation of crude extracts and protein purification}

All steps were carried out at $4^{\circ} \mathrm{C}$ under an atmosphere of $\mathrm{N}_{2}$ and $1 \% \mathrm{H}_{2}$ in an anaerobic chamber (Coy Laboratories), unless stated otherwise. Wet cell paste of MC4100 (DE3) transformed cells containing Twin-Strep-tagged HypC in complex with HypD was suspended at a ratio of 1:5 $(\mathrm{m} / \mathrm{v})$ in breaking buffer (100 mM Tris- $\mathrm{HCl} \mathrm{pH} 8,150 \mathrm{mM} \mathrm{NaCl}, 10 \mathrm{mM} \mathrm{Mg}$ acetate, $5 \mathrm{mg} / \mathrm{l}$ DNase, and $0.2 \mathrm{mM}$ PMSF). Ice-cooled cells were disrupted by sonication $(70 \mathrm{~W}$ for $30 \mathrm{~min}$ with $0.5 \mathrm{~s}$ pulses). Unbroken cells and debris were removed by centrifugation for $30 \mathrm{~min}$ at $15000 \mathrm{~g}$ at $4^{\circ} \mathrm{C}$. The supernatant or crude extract derived from $40-60 \mathrm{~g}$ of wet cells was used for anaerobic purification of the HypCD complex. Proteins were isolated by chromatography on a $20 \mathrm{ml}$ Strep-Tactin-XT column (IBA) using gravity-flow. Unbound proteins were removed by

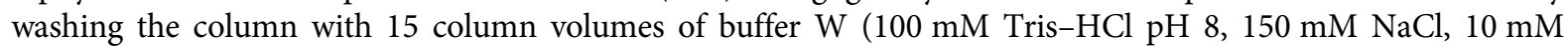
magnesium acetate, and $1 \%$ glycerol). Recombinant protein was eluted with the same buffer but including $50 \mathrm{mM}$ biotin. The protein was desalted on a Sephadex G-25 column (GE Healthcare) in buffer W. Purified protein was concentrated by centrifugation at $5000 \mathrm{~g}$ using centrifugal filters (Amicon Ultra, $30 \mathrm{kDa}$ cut-off, Millipore) and applied to a Superdex 200 gel filtration column $(2.6 \times 60 \mathrm{~cm})$ equilibrated with the same buffer.

The HypCD complex was adjusted to $40-50 \mathrm{mg} / \mathrm{ml}$ for spectroscopy $(1-2 \mathrm{mg} / \mathrm{ml}$ for UV/Vis spectroscopy). Such protein preparations are colored (reddish-brown at $>10 \mathrm{mg} / \mathrm{ml}$ ) and referred to 'as-isolated'. Reduction was achieved in the presence of $1-10 \mathrm{mM} \mathrm{NaDT}$ and oxidation was achieved with 20 or 40 eq. phenazine methosulfate (PMS) or $0.2-2 \% \mathrm{v} / \mathrm{v} \mathrm{H}_{2} \mathrm{O}_{2}$. To 'recover' the as-isolated state of the HypCD complex, NaDT-, PMS- or $\mathrm{H}_{2} \mathrm{O}_{2}$-containing buffer was exchanged using Viva-spin centrifugal concentrators ( $30 \mathrm{kDa}$ MWCO). The protein sample was diluted $120 \times$ with protein buffer at a $\mathrm{pH} 8,9$, or 10 (as indicated) and concentrated at $4000 \mathrm{~g}$ for $30 \mathrm{~min}$. The concentrated sample was diluted $120 \times$ again with fresh protein buffer and re-adjusted to the original concentration of $40-50 \mathrm{mg} / \mathrm{ml}$.

His-tagged HypF and Strep-tagged HypE were purified from BL21(DE3) cells transformed with the appropriate plasmid derivative as described previously $[12,19]$. A novel protocol was developed for reconstitution of a homogenous HypEF complex on an affinity column ensuring that the HypEF complex is 'saturated' with its interaction partner. The Strep-tagged HypE protein was loaded on a Strep-Tactin gravity flow column. After washing with three column volumes of buffer W, His-HypF was loaded in two-fold molar excess, followed by three washing steps with the same buffer. HypEF complex was eluted with buffer W containing $50 \mathrm{mM}$ biotin. The eluted protein was collected and loaded on a gel filtration column equilibrated with buffer $\mathrm{W}$ containing $1 \mathrm{mM} \mathrm{NaDT}$ and $5 \mathrm{mM}$ DTT. The HypEF complex was eluted in one major peak.

\section{Polyacrylamide gel electrophoresis and metal quantification}

To check for protein purity, 10-20 $\mu \mathrm{g}$ of the HypE, HypCD or HypEF complexes were separated by SDSPAGE (NuPAGE 4 to 20\% Tris-glycine, Invitrogen). Protein identity was confirmed by protein mass spectrometry as described previously [29]. A defined amount of crude extracts derived from a HypD deletion mutant (DHP-D) [30] that was complemented with pT-hypDEFC ${ }^{\text {StrepXT }}$ plasmid was analyzed for hydrogenase activity via native PAGE (NuPAGE 4-12\% bis-tris glycine) under non-denaturing conditions. Protein samples were incubated with $4 \%(\mathrm{w} / \mathrm{v})$ Triton X-100 prior to application to the gels. The gel was subsequently stained for hydrogenase enzyme activity as reported previously [31]. The protein concentration was determined as described by Lowry and co-workers [32]. Metal analysis was done by inductively coupled plasma mass spectrometry (ICP-QqQ-MS) using a high resolution 8800 ICP-QqQ-MS device (Agilent Technologies) as described previously [33]. The instrument was run with $\mathrm{H}_{2}$ as reduction gas in the Octopole Reaction System to reduce polyatomic interferences and in direct infusion mode via a nebulizer. ICP multi-element standard solution XVI (Merck) and rhodium was used as internal standards.

\section{UV/Vis spectroscopy}

Absorption spectra were recorded under anaerobic conditions in septum-sealed $1 \mathrm{~mm}$ quartz cuvettes at room temperature $(\sim 300 \mathrm{~K})$, using a Shimadzu UV-2600 spectrophotometer. The protein solution was diluted to 1$2 \mathrm{mg} / \mathrm{ml}$. Spectra of reduced HypCD (with $1 \mathrm{mM} \mathrm{NaDT}$ ) or oxidized HypCD (with $0.2 \% \mathrm{H}_{2} \mathrm{O}_{2}$ ) were recorded 
until no further changes were observed (Supplementary Figure S2). Due to the intense color of PMS, no UV/ Vis spectra of PMS-oxidized HypCD were recorded.

\section{Resonance Raman and FTIR spectroscopy}

As-isolated, reduced (with $10 \mathrm{mM} \mathrm{NaDT}$ ), oxidized (with $2 \% \mathrm{H}_{2} \mathrm{O}_{2}$ ), and 'recovered' samples of HypCD (40$50 \mathrm{mg} / \mathrm{ml}$ ) were prepared as described above. For each sample, $5 \mu$ l protein solution were squeezed between two glass coverslips using a $0.5 \mathrm{~mm}$ spacer and sealed with vacuum grease to avoid dehydration and contact with air. Resonance Raman (RR) spectroscopy was performed at room temperature ( 300 K) on a LabRAM spectrometer (HORIBA, Kyoto, Japan) as described previously [34] but using a frequency-stabilized diodepumped solid-state laser $(\mathrm{CNI})$ emitting at $457 \mathrm{~nm}$. Laser power at the sample was $\sim 5 \mathrm{~mW}$. The recorded spectra were baseline corrected using the rubber band method implemented in the OPUS software and normalized to the amide I vibration at $1670 \mathrm{~cm}^{-1}$ (Supplementary Figure S3). Due to the intense color of PMS, no RR spectra of PMS-oxidized HypCD were recorded.

Attenuated total reflection (ATR) Fourier-transform infrared (FTIR) spectroscopy was performed in an anaerobic gas chamber (Coy Laboratories) in a continuously purged and effectively water-free atmosphere of $\mathrm{N}_{2}$ and $1 \% \mathrm{H}_{2}$. All spectroscopic experiments were performed at room temperature $(\sim 300 \mathrm{~K})$ on a Tensor27 FTIR spectrometer (Bruker Optik) equipped with a three-reflection ZnSe/silicon crystal ATR cell (Smiths Detection) as described previously [35]. Beam path, spectrometer, and anaerobic chamber were purged with dry $\mathrm{N}_{2}$ gas as provided by an Inmatec nitrogen generator. For each experiment, $1 \mu \mathrm{l}$ of HypCD protein sample (40-50 mg/ml, as-isolated, NaDT-reduced, oxidized with $\mathrm{H}_{2} \mathrm{O}_{2}$ or PMS, and 'recovered' as indicated above) was pipetted onto the silicon crystal, dried under $\mathrm{N}_{2}$, and re-hydrated in the presence of an aerosol by running the gas mixture through a wash bottle with $A$. bidest $[35,36]$. Absorbance spectra were recorded with a spectral resolution of $2 \mathrm{~cm}^{-1}$ and up to 10.000 interferometer scans at $80 \mathrm{kHz}$ scanner velocity.

\section{EPR and Mössbauer spectroscopy}

For electron paramagnetic resonance (EPR) spectroscopy different preparations of the HypCD complex were prepared $\left(40-50 \mathrm{mg} / \mathrm{ml}\right.$, as-isolated, NaDT-reduced, oxidized with $\mathrm{H}_{2} \mathrm{O}_{2}$ or PMS, and 'recovered' as indicated above). Data were recorded with a Bruker Elexsys E580 X-band spectrometer, equipped with an Oxford Instruments ESR900 helium flow cryostat or Bruker ER 167FDS-Q liquid nitrogen finger dewar. A modulation amplitude of $1.0 \mathrm{mT}$ at a frequency of $100 \mathrm{kHz}$ was used.

For Mössbauer spectroscopy, different preparations of the ${ }^{57} \mathrm{Fe}$-labeled HypCD complex were prepared (40-50 mg/ml, as-isolated, NaDT-reduced, oxidized with $\mathrm{H}_{2} \mathrm{O}_{2}$ or PMS). Mössbauer spectra were recorded in the constant acceleration mode with a conventional spectrometer from Wissel $\mathrm{GmbH}$ with a multi-channel analyzer in the time-scale mode (WissEL GmbH). Experiments at $77 \mathrm{~K}$ were conducted with an $\mathrm{LN}_{2}$ bath cryostat (Oxford Instruments). High-field, low temperature spectra were measured with the same type of spectrometer in a helium closed-cycle cryostat equipped with a superconducting magnet (CRYO Industries of America Inc.) operating with the applied field parallel to the $\gamma$-rays. Isomer shifts $\delta$ are given relative to $\alpha$-iron at room temperature. Data were transferred from the multi-channel analyzer to a PC for analysis employing the public domain program Vinda running on an Excel 2003 platform. Magnetically split spectra were simulated with the spin Hamiltonian formalism. Spectra were analyzed by least-squared fits using Lorentzian line shapes.

\section{Microscale thermophoresis}

For protein labeling and MST [37] measurements, $100 \mathrm{mM}$ Tris- $\mathrm{HCl}(\mathrm{pH}$ 8) containing $150 \mathrm{mM} \mathrm{NaCl}$, $10 \mathrm{mM} \mathrm{MgCl} 2,1 \%$ glycerol, and $0.05 \%$ Tween 20 was used. Experiments were performed on a Monolith NT.115 (NanoTemper Technologies). The protein sample was labeled using the companys' protein labeling kit RED-NHS. The labeling reaction was performed according to the manufacturers' instructions applying a concentration of $40 \mu \mathrm{M}$ of HypCD complex (molar dye:protein ratio $\approx 3: 1)$ at room temperature $(\sim 300 \mathrm{~K})$ for $30 \mathrm{~min}$ in the dark. Unreacted dye was removed with the supplied dye removal column. The presence of the $[\mathrm{Fe}](\mathrm{CN})_{2} \mathrm{CO}$ moiety on the dye-treated HypCD complex was routinely verified by FTIR. Labeled HypCD complex was adjusted to $40 \mathrm{nM}$ with the buffer mentioned above. Then, a series of 16 consecutive $1: 1$ dilutions of the HypE or the HypEF complex ('ligand') was prepared using the same buffer, producing ligand concentrations ranging from $10 \mathrm{nM}$ to $1000 \mu \mathrm{M}$. For the measurement, each ligand dilution was mixed with one volume of labeled HypCD complex $(40 \mathrm{nM})$. After $20 \mathrm{~min}$ incubation followed by centrifugation at $20000 \mathrm{~g}$ for $15 \mathrm{~min}$, the samples were loaded onto standard capillaries. Monolith instrument parameters were adjusted to 
40-60\% LED power and 90-100\% MST power. Data of at least three independent measurements were analyzed by NanoTemper analysis software. MST signals with a 'laser-on' time of $30 \mathrm{~s}$ and a 'laser-off time of $5 \mathrm{~s}$ were applied for analysis. For affinity measurements under anaerobic conditions, in the presence of $1 \mathrm{mM} \mathrm{NaDT}$, all samples and dilution series were prepared anaerobically in a COY anaerobic chamber.

\section{Results}

\section{Production of the HypCD complex and incorporation of ${ }^{57} \mathrm{Fe}$}

To produce the mature HypCD complex with complete cluster and cofactor content in preparative amounts, an E. coli expression system and an optimized purification protocol were developed. For overproduction of the recombinant HypCD complex, we constructed the plasmid pT-hypDEFC ${ }^{\text {StrepXT }}$ with two Strep-tag sequences fused to HypC. The Twin-Strep-tag has much higher affinity to Strep-Tactin-XT resins than the single Strep-tag and thus enabling efficient purification of the HypCD complex with high purity and structural integrity [38]. To confirm the catalytic competence of the pT-hypDEFC ${ }^{\text {StrepXT }}$ product for its ability to restore the hydrogenase activity in vivo, the plasmid was introduced into a hypD deletion mutant (DHP-D) and the restored hydrogenase activity was confirmed by specific staining after separation of the enzyme by native-PAGE. The stained bands corresponding to active [NiFe]-hydrogenases from E. coli Hyd-1, Hyd-2, and Hyd-3 are indicated in Figure 2A. Furthermore, we optimized the growth conditions to obtain high yields of the mature HypCD complex (Supplementary Figure S1). Several HypCD preparations were isolated from transformed cells grown in different media. Protein preparations were routinely probed by SDS-PAGE (Figure 2B) and ATR FTIR spectroscopy to identify the $[\mathrm{Fe}](\mathrm{CN})_{2} \mathrm{CO}$ cofactor precursor (data not shown). We found that both media composition and growth conditions are important for cofactor integrity and protein yield. High yields of partially mature protein were obtained using full medium (TGYEP, $1 \mathrm{~g}$ wet cells with $\sim 12 \mathrm{mg}$ recombinant protein) while M9 minimal medium delivered lower yields but fully functional protein $(1 \mathrm{~g}$ wet cells contained $\sim 4 \mathrm{mg}$ recombinant protein). Metal analysis of the mature HypCD complex performed by ICP-MS revealed that protein preparations typically contain five equivalents of iron per protein molecule. The amount of nickel, copper, and zinc were not significant (Table 1). For Mössbauer spectroscopy, two growth media were used to produce the ${ }^{57} \mathrm{Fe}$-labeled HypCD complex. Both, M9* and TGYEP ${ }^{\star}$ allowed good growth rates (Supplementary Figure S1) and delivered similar yields with a ${ }^{57} \mathrm{Fe}$ enrichment of more than $99 \%$. For this, a

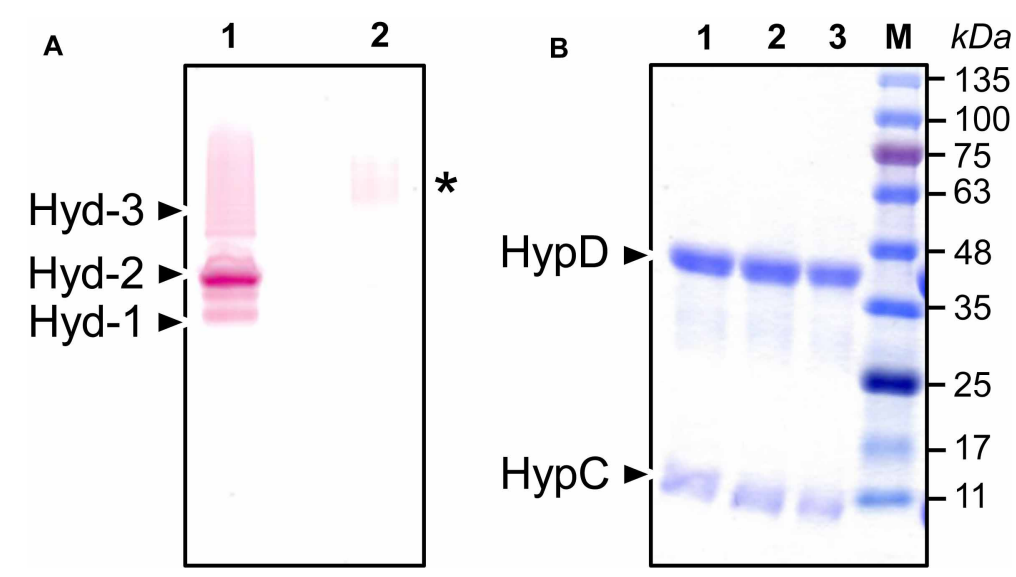

Figure 2. Production of the functional HypCD complex.

(A) Native PAGE confirms the catalytic competence of HypCD. Strain DHP-D $(\Delta h y p D)$ can be complemented in trans by


non-denaturing conditions and stained for hydrogenase activity. Lane 1: complemented DHP-D strain. Lane 2: DHP-D strain. The stained bands corresponding to active Hyd-1, Hyd-2 and Hyd-3 are indicated. A weak hydrogenase-independent activity due to formate dehydrogenase (Fdh) acts as a loading control (*). (B) SDS-PAGE confirms the purity of the strep-tagged HypCD preparations (7 $\mu \mathrm{g}$ of each) from cells grown in different media. M9* (lane 1) and TGYEP* (lane 2) delivered similar yields of fully charged HypCD. TGYEP full medium (lane 3) delivered higher yields but only partially charged HypCD. M, $6 \mu$ l of PS10 Plus pre-stained marker. 
Table 1 Metal contents of HypCD preparations from cells grown in different media

\begin{tabular}{|c|c|c|c|c|}
\hline & \multicolumn{4}{|c|}{ mol metal/mol HypCD } \\
\hline & \multicolumn{2}{|l|}{$20 \mu M^{57} \mathrm{Fe}$} & \multicolumn{2}{|l|}{$100 \mu M^{57} \mathrm{Fe}$} \\
\hline & $\overline{\text { M9* }}$ & TGYEP* & TGYEP & TGYEP* \\
\hline${ }^{57} \mathrm{Fe}$ & $\begin{array}{l}4.88 \pm 0.31(n=5) \\
(99.7 \%)\end{array}$ & $\begin{array}{l}4.59 \pm 0.20(n=5) \\
(49.3 \%)\end{array}$ & $\begin{array}{l}4.38 \pm 0.18(n=5) \\
(50.3 \%)\end{array}$ & $\begin{array}{l}4.79 \pm 0.22(n=5) \\
(99.5 \%)\end{array}$ \\
\hline $\mathrm{Ni}$ & $0.002(n=4)$ & $0.001(n=4)$ & $\mathrm{ND}(n=3)$ & $\mathrm{ND}(n=3)$ \\
\hline Zn & $0.002(n=3)$ & $0.002(n=3)$ & $0.001(n=3)$ & $\mathrm{ND}(n=3)$ \\
\hline $\mathrm{Cu}$ & $0.0003(n=3)$ & $\mathrm{ND}(n=3)$ & $\mathrm{ND}(n=3)$ & $0.0002(n=3)$ \\
\hline
\end{tabular}

The metal content was quantified by ICP-MS. The molar ratio is calculated based on the molecular mass of the HypCD complex (50 kDa). Values in brackets represent percentage of ${ }^{57} \mathrm{Fe}$-enrichment. All samples were measured with two independent technical replicates. Data are averages of at least three independent determinations $(n)$ and reported as the mean \pm SD (standard deviation). ND, not detected.

concentration of $20 \mu \mathrm{M}$ and $100 \mu \mathrm{M}{ }^{57} \mathrm{Fe}$ were necessary in the $\mathrm{M}^{*}$ and $\mathrm{TGYEP}^{*}$ media, respectively. Only $\sim 50 \%$ enrichment of ${ }^{57} \mathrm{Fe}$ was detected when $20 \mu \mathrm{M}{ }^{57} \mathrm{Fe}$ were added to $\mathrm{TGYEP}^{*}$ medium or when the cells were grown in TGYEP full medium (Table 1). Thus, a full enrichment of the ${ }^{57} \mathrm{Fe}$-labeled HypCD complex can be achieved with five-fold lower ${ }^{57} \mathrm{Fe}$ concentration by using our chemically defined $\mathrm{M} 9^{*}$ minimal medium, suggesting that the approaches developed herein can be generally applied for ${ }^{57} \mathrm{Fe}$ enrichment of other metalloproteins.

\section{The redox changes of HypCD are reversible}

A dilute solution of the HypCD scaffold complex in the 'as-isolated' state (see above) showed the typical UV/ Vis absorbance band at $410 \mathrm{~nm}$ characteristic for iron-sulfur proteins (Figure 3A) [39]. Resonance Raman spectroscopy confirmed the presence of a $[4 \mathrm{Fe}-4 \mathrm{~S}]^{+2}$ cluster (Figure 3B) [40]. Incubation of HypCD with $0.2 \%$ $\mathrm{H}_{2} \mathrm{O}_{2}$ or $1 \mathrm{mM} \mathrm{NaDT}$ led to a sudden decrease in absorbance hinting at an oxidation or reduction event, respectively. We found the effect of $\mathrm{H}_{2} \mathrm{O}_{2}$ to be irreversible in a previous study [25]; however, upon buffer exchange it was possible to restore the 'as-isolated state'. While a mild re-reduction with dithiothreitol (DTT) was necessary to recover the UV/Vis and RR spectra of $\mathrm{H}_{2} \mathrm{O}_{2}$-treated $\mathrm{HypCD}$, removal of $\mathrm{NaDT}$ immediately restored its absorption properties (Supplementary Figures S2, S3). These results suggest a qualitatively reversibly
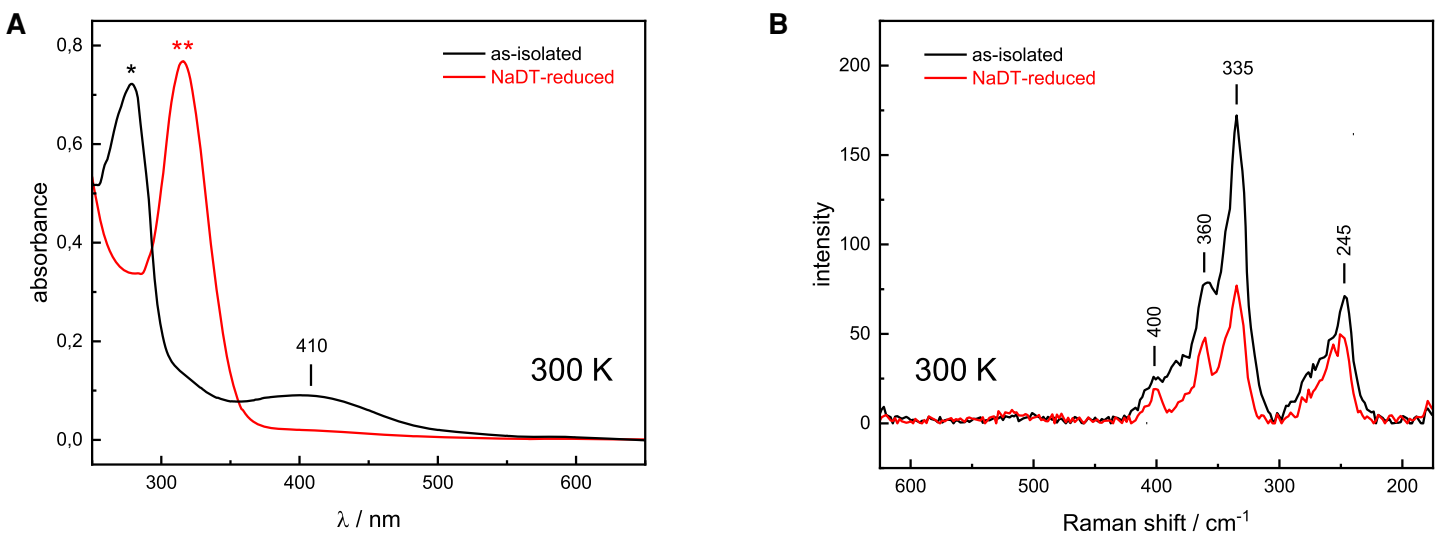

Figure 3. UV/Vis and Raman spectroscopy.

(A) UV/Nis spectra of the HypCD scaffold complex at pH 9 in as-isolated form (black) and in the presence of $1 \mathrm{mM} \mathrm{NaDT}$ (red). Note the decrease in the [4Fe-4S] cluster band at $410 \mathrm{~nm}$ upon reduction. UVA bands include $280 \mathrm{~nm}$ (* to protein) and $315 \mathrm{~nm}$ (** to NaDT). (B) Resonance Raman spectra of the HypCD complex at pH 9 in as-isolated form (black) and in the presence of $10 \mathrm{mM} \mathrm{NaDT}$ (red). Note the decrease in the terminal $\left(400\right.$ and $\left.360 \mathrm{~cm}^{-1}\right)$ and bridging Fe-S bands $\left(335\right.$ and $\left.245 \mathrm{~cm}^{-1}\right)$ upon reduction. These feature confirm the assignment of a $[4 \mathrm{Fe}-4 \mathrm{~S}]^{2+}$ cluster on as-isolated HypCD. 
A

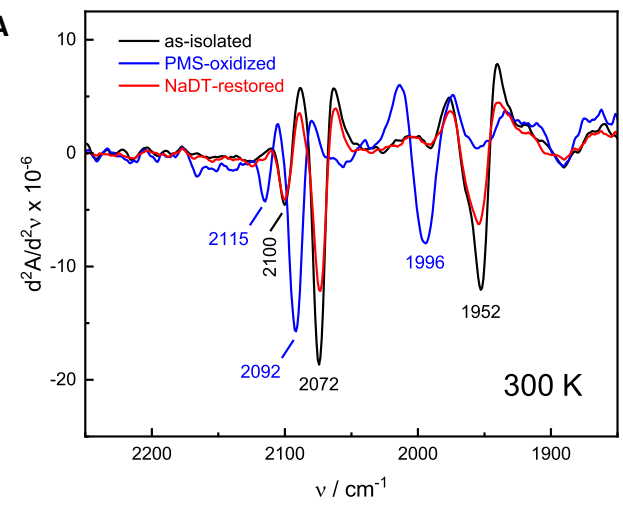

B

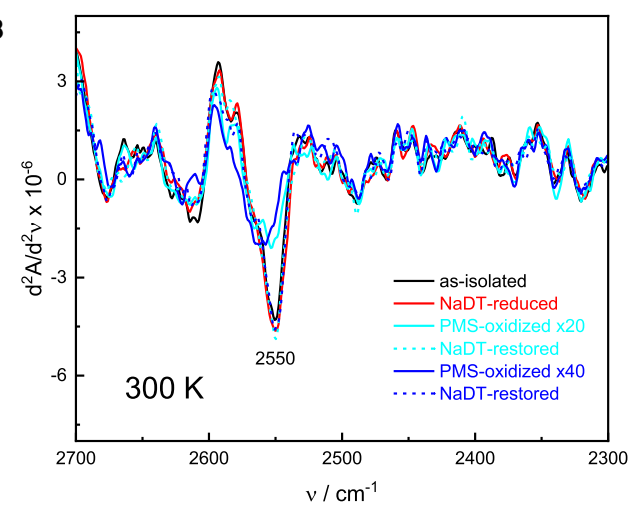

Figure 4. ATR FTIR spectroscopy.

(A) Second derivative ATR FTIR spectra of the HypCD complex in as-isolated form (black), in the presence 40 eq. PMS (blue), or restored with $10 \mathrm{mM} \mathrm{NaDT}$ after reduction (red). Note the shift of the $\mathrm{CO} / \mathrm{CN}^{-}$bands to higher frequencies upon oxidation with PMS, that hints at oxidation of the cofactor precursor from $[\mathrm{Fe}]^{2+}$ to $[\mathrm{Fe}]^{3+}$. As-isolated and NaDT-restored samples show the same band positions. (B) As-isolated (black) and NaDT-reduced HypCD (red) shows a clear band at $2550 \mathrm{~cm}^{-1}$ that can be assigned to $\mathrm{SH}$ vibration of cysteine residues. In the presence of 20 or 40 eq. PMS the absorbance decreased (cyan and blue traces), indicative of disulfide bond formation. This process is reversible, as highlighted by the recovery of the SH signal in NaDT-restored sample (dashed traces).

conversion of redox states, including a NaDT-reduced and $\mathrm{H}_{2} \mathrm{O}_{2}$-oxidized iron-sulfur cluster. EPR spectra of the $\mathrm{H}_{2} \mathrm{O}_{2}$-incubated sample revealed a $[3 \mathrm{Fe}-4 \mathrm{~S}]^{+}$species (Supplementary Figure S4) that has been discussed as oxidative damage to the cluster previously [26]. Therefore, we screened for an alternative oxidant. Investigating the $[\mathrm{Fe}](\mathrm{CN})_{2} \mathrm{CO}$ cofactor precursor by ATR FTIR spectroscopy we observed similar band shifts in the presence of $\mathrm{H}_{2} \mathrm{O}_{2}$ and PMS (Figure 4 and Supplementary Figures S5, S6). Second derivative spectra of HypCD reveal the typical $\mathrm{CO} / \mathrm{CN}^{-}$signature of the cofactor precursor in the 'as-isolated' state $\left(2100,2072\right.$, and $\left.1952 \mathrm{~cm}^{-1}\right)$. Oxidation with 40 eq. PMS (blue traces in Figure 4A) resulted in a shift to higher frequencies $(2115,2092$, and $\left.1996 \mathrm{~cm}^{-1}\right)$, indicative of an oxidized $[\mathrm{Fe}](\mathrm{CN})_{2} \mathrm{CO}$ cofactor precursor [24,27]. The effect of PMS and $\mathrm{H}_{2} \mathrm{O}_{2}$ was found to be reversible after buffer exchange and reduction, similar to what was observed by UV/Vis and RR spectroscopy (Supplementary Figures S2, S3). The intense $\mathrm{CO}_{2}$ band that hints at oxidative damage in the presence of $\mathrm{H}_{2} \mathrm{O}_{2}$ was not observed with PMS (Supplementary Figure S4). Moreover, we noted a pronounced decline of SH signals [41] in $\mathrm{H}_{2} \mathrm{O}_{2^{-}}$and PMS-oxidized sample at $2550 \mathrm{~cm}^{-1}$ compared with as-isolated, NaDT-reduced, and NaDT-restored preparations (Figure 4B and Supplementary Figure S6B). This is likely to reflect disulfide bond formation under oxidizing conditions.

Summing up, our analysis of the HypCD scaffold complex suggests the existence of at least three redox states. We assume that the iron-sulfur cluster may interconvert between $[4 \mathrm{Fe}-4 \mathrm{~S}]^{+2}$ (as-isolated) and $[4 \mathrm{Fe}-4 \mathrm{~S}]^{+}$ (NaDT-reduced) whereas the mononuclear iron center may change between $[\mathrm{Fe}]^{2+}$ (as-isolated) and $[\mathrm{Fe}]^{3+}$ (PMS-oxidized). Additionally, EPR spectroscopy identified an 'overoxidized' [3Fe-4S $]^{+}$cluster in some preparations, which hints at damaged HypD. In order to gain direct evidence for the existence of intact reduced and oxidized metal centers, we performed further experiments.

\section{Characterization of the reduced and oxidized metal centers}

The $[4 \mathrm{Fe}-4 \mathrm{~S}]^{2+}$ cluster of the HypCD scaffold complex is diamagnetic and therefore EPR-inactive. In an earlier attempt to characterize the HypD monomer by Blokesch and colleagues, traces of a $[3 \mathrm{Fe}-4 \mathrm{~S}]^{+}$cluster were detected [26], in agreement with our data (Supplementary Figure S4). While these signals vanished upon reduction with $50 \mathrm{mM} \mathrm{NaDT}$ at $\mathrm{pH} 8$, no $\mathrm{S}=1 / 2$ or high spin EPR signal of the reduced $[4 \mathrm{Fe}-4 \mathrm{~S}]^{+}$cluster was observed [26]. Being aware that the redox potential of $\mathrm{NaDT}$ is $\mathrm{pH}$-dependent [42], we adjusted the sample to $\mathrm{pH} 10$ and probed the HypCD complex in the absence and presence of $10 \mathrm{mM} \mathrm{NaDT}$ (Figure 5A). X-band EPR spectroscopy at low temperature now revealed a sharp rhombic EPR signal with $g$-values 2.062, 1.935, and 1.911 , which is typical for a [4Fe-4S $]^{+}$cluster [43]. Only minor reduction was observed at $\mathrm{pH} 8$. Moreover, we investigated a sample of the HypCD complex in the presence of 40 eq. PMS (Figure 5A). Only an intense 

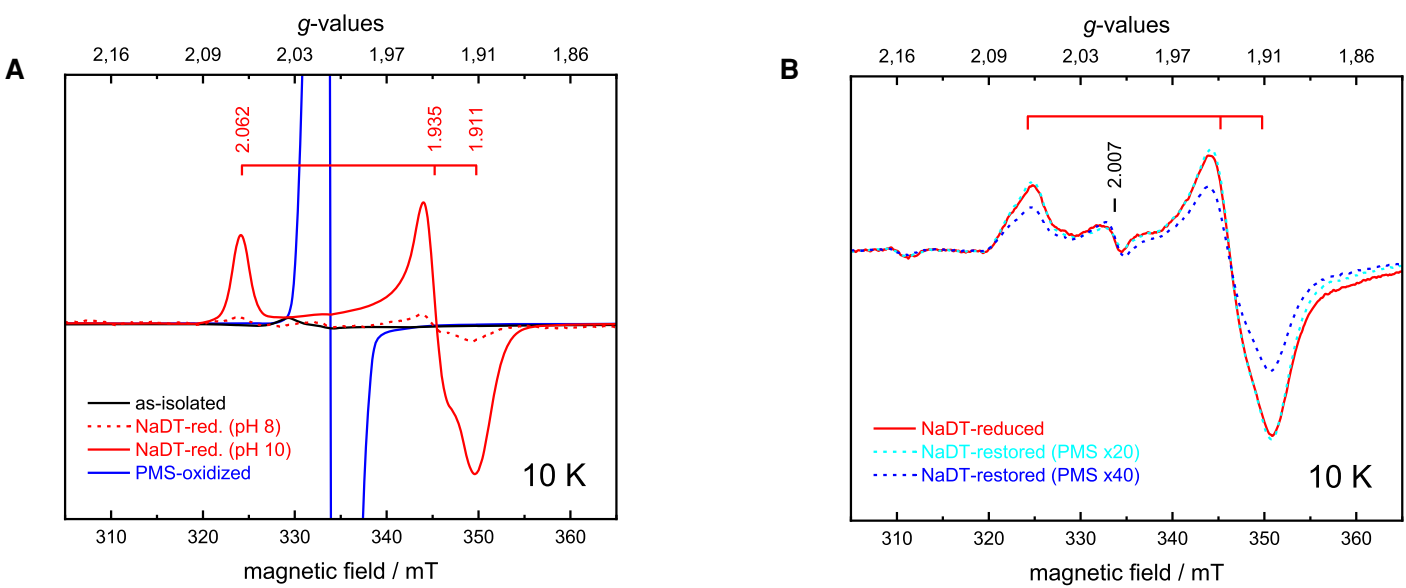

Figure 5. EPR spectroscopy.

(A) X-band EPR spectra at $10 \mathrm{~K}$ of the HypCD complex in as-isolated form (diamagnetic, black) and NaDT-reduced form at $\mathrm{pH}$ 8 (dashed red) and pH 10 (red). The $g$-values of the reduced [4Fe-4S] cluster are annotated. In the presence of PMS, only the phenanzine free radical is observed (blue). (B) Comparison of NaDT-reduced (red trace) and NaDT-restored HypCD (dashed traces) at $\mathrm{pH}$ 9. The former was reduced from as-isolated sample directly. The later was treated with PMS (20 or 40 eq.), washed, and reduced with $\mathrm{NaDT}$ to restore the $[4 \mathrm{Fe}-4 \mathrm{~S}]^{+}$state. The signal at $g=2.007$ hints at a dithionite radical.

sharp isotropic signal, which under the conditions employed for the detection of Fe/S EPR signals does not show hyperfine couplings due to the modulation amplitude presumably from PMS radicals [44] was detected. No traces of iron-sulfur clusters like $[3 \mathrm{Fe}-4 \mathrm{~S}]^{+}$or $[4 \mathrm{Fe}-4 \mathrm{~S}]^{3+}$ were observed $[26,45]$. This confirms the utility of PMS as a site-selective oxidant of the mononuclear iron center (Figure 4A) and suggests that the 'overoxidized' iron-sulfur cluster is biologically not relevant. The lack of damage to the [4Fe- $4 \mathrm{~S}]$ cluster in the presence of PMS is additionally highlighted in Figure $5 \mathrm{~B}$. Here, the $[4 \mathrm{Fe}-4 \mathrm{~S}]^{+}$spectrum of $\mathrm{HypCD}$ after direct reduction (red) is compared with the $[4 \mathrm{Fe}-4 \mathrm{~S}]^{+}$spectrum after PMS-mediated oxidation and re-reduction (dashed traces). With 20 eq. of PMS, full recovery was observed.

For the first time, EPR spectroscopy identified the reduced form of the iron-sulfur cluster on the HypCD scaffold complex, corroborating its redox activity. However, while ATR FTIR spectroscopy suggested at least two possible redox states of the $[\mathrm{Fe}](\mathrm{CN})_{2} \mathrm{CO}$ cofactor precursor (Figure 4), no direct identification of its redox states has been obtained yet. To provide a comprehensive description of all metal sites on the functional HypCD complex, we enriched the protein in ${ }^{57} \mathrm{Fe}$ and performed Mössbauer spectroscopy in the temperature range from $4.2-200 \mathrm{~K}$ and within an external $B$-field of 0.0-5.0 T (Supplementary Figures S7, S8). Figure 6A shows the Mössbauer spectrum of the as-isolated HypCD complex at $77 \mathrm{~K}$ and $0 \mathrm{~T}$, revealing the presence of two distinct species. The main part of the spectrum is a quadrupole doublet (black traces) comprised of two components each representing a $\mathrm{Fe}^{2.5+}-\mathrm{Fe}^{2.5+}$ pair of a $[4 \mathrm{Fe}-4 \mathrm{~S}]^{2+}$ cluster [46]. Mössbauer spectra from 4.2$200 \mathrm{~K}$ (Supplementary Figure S8) show a temperature dependent quadrupole splitting of the valence delocalized $\mathrm{Fe}^{2.5+}-\mathrm{Fe}^{2.5+}$ pairs of the [4Fe-4S] cluster as observed by Middleton and colleagues [47] and subsequently reported for multiple [4Fe-4S] clusters [48]. While our data are in excellent agreement with the Mössbauer spectra of HypCD reported by Blokesch and colleagues [26], an additional component was included in the description, accounting for a shoulder at $\sim-0.5 \mathrm{~mm} / \mathrm{s}$ (magenta trace). The assignment of the $[\mathrm{Fe}](\mathrm{CN})_{2} \mathrm{CO}$ cofactor is in accordance with a similar species in the [NiFe]-hydrogenase of Ralstonia eutropha [49]. Mössbauer spectroscopy in an external $B$-field of $5.0 \mathrm{~T}$ (Figure 6B) confirms the diamagnetic state of the $[4 \mathrm{Fe}-4 \mathrm{~S}]^{2+}$ cluster and identifies the mononuclear iron site as a diamagnetic low-spin $[\mathrm{Fe}]^{2+}$ with $\delta=0.22 \mathrm{~mm} / \mathrm{s}$ and $\Delta \mathrm{E}_{\mathrm{Q}}=1.62 \mathrm{~mm} / \mathrm{s}$. Upon addition of $10 \mathrm{mM} \mathrm{NaDT}$, the $[4 \mathrm{Fe}-4 \mathrm{~S}]^{2+}$ cluster was reduced to a paramagnetic $[4 \mathrm{Fe}-4 \mathrm{~S}]^{+}$cluster (Figure $6 \mathrm{C}, \mathrm{D}$ ), whereas the $[\mathrm{Fe}](\mathrm{CN})_{2} \mathrm{CO}$ moiety showed no detectable changes. The two components representing the $[4 \mathrm{Fe}-4 \mathrm{~S}]^{+}$cluster (red traces) can be assigned to a $\mathrm{Fe}^{2.5+}-\mathrm{Fe}^{2.5+}$ pair $(\delta=0.43 \mathrm{~mm} /$ $\mathrm{s}$ and $\left.\Delta \mathrm{E}_{\mathrm{Q}}=1.10 \mathrm{~mm} / \mathrm{s}\right)$ and a $\mathrm{Fe}^{2+}-\mathrm{Fe}^{2+}$ pair $\left(\delta=0.59 \mathrm{~mm} / \mathrm{s}\right.$ and $\left.\Delta \mathrm{E}_{\mathrm{Q}}=1.88 \mathrm{~mm} / \mathrm{s}\right)$. No oxidation of the $[4 \mathrm{Fe}-4 \mathrm{~S}]^{2+}$ cluster was observed in the presence of 40 eq. PMS (Figure $6 \mathrm{E}, \mathrm{F}$ ), in agreement with our EPR data. However, in an external $B$-field of $5.0 \mathrm{~T}$, we were able to identify a component that resembles a high-spin $[\mathrm{Fe}]^{3+}$ site $\left(\delta=0.26 \mathrm{~mm} / \mathrm{s}\right.$ and $\left.\Delta \mathrm{E}_{\mathrm{Q}}=0.81 \mathrm{~mm} / \mathrm{s}\right)$. The Mössbauer parameters are summarized in Table 2 . 

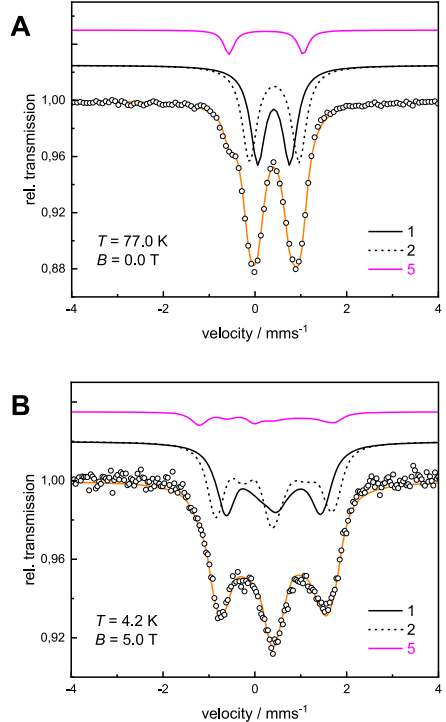

C

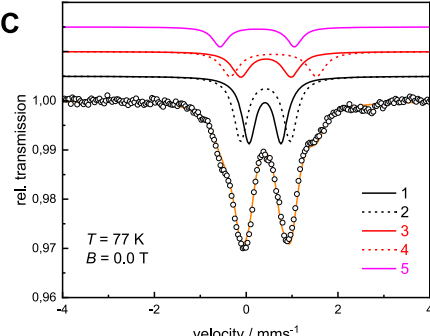

D



E


Figure 6. Mössbauer spectroscopy.

Spectra of the ${ }^{57} \mathrm{Fe}-$ labeled HypCD complex (40-50 mg/ml at pH 9) in as-isolated (A and B), NaDT-reduced (C and D), and PMS-oxidized form (E and F) obtained at $77 \mathrm{~K}(\mathbf{A}, \mathbf{C}, \mathbf{E})$ or $4.2 \mathrm{~K}$ and an external $B$-field or $5.0 \mathrm{~T}$ (B,D,F). Legend: experimental data (open circles), sum of simulations (orange trace), $[4 \mathrm{Fe}-4 \mathrm{~S}]^{2+}\left(1+2\right.$, black), $[4 \mathrm{Fe}-4 \mathrm{~S}]^{+}(3+4, \mathrm{red}),[\mathrm{Fe}]^{2+}(5$, magenta), and $[\mathrm{Fe}]^{3+}$ (6, blue). See Table 2 for further details.

\section{The affinity between HypCD and $\operatorname{HypE}(\mathrm{F})$ dependents on the redox state}

MicroScale thermophoresis was applied to determine the binding affinities of the HypCD complex to its in vivo interaction partner HypE. The concentration of dye-labeled HypCD complex was kept constant (40 nM) and mixed with increasing concentrations of the binding partner HypE. The binding affinities were determined under aerobic or reducing conditions $(1 \mathrm{mM} \mathrm{NaDT})$. Figure 7A depicts the HypE dependence of the MST signal for dye-labeled HypCD. The MST signals were fitted according to Michaelis-Menten kinetics to obtain the apparent binding affinity between HypCD and HypE (solid lines). The data show that HypCD displayed a higher binding affinity to HypE under reducing conditions $\left(K_{\mathrm{d}}=4.67 \pm 1.5 \mu \mathrm{M}\right)$ compared with aerobic

Table 2 Mössbauer parameters

\begin{tabular}{|c|c|c|c|c|c|c|c|c|c|c|c|c|c|c|}
\hline \multirow{3}{*}{$\begin{array}{l}\text { Metal center } \\
\text { Subspectrum }\end{array}$} & \multicolumn{6}{|c|}{$[4 \mathrm{Fe}-4 \mathrm{~S}]^{2+}$} & \multicolumn{6}{|c|}{$\left[_{4 \mathrm{Fe}-4 \mathrm{~S}}\right]^{1+}$} & \multicolumn{2}{|l|}{ [Fe] } \\
\hline & \multicolumn{3}{|c|}{$\mathrm{Fe}^{2.5+}-\mathrm{Fe}^{2.5+}$} & \multicolumn{3}{|c|}{$\mathrm{Fe}^{2.5+}-\mathrm{Fe}^{2.5+}$} & \multicolumn{3}{|l|}{$\mathrm{Fe}^{2+}$} & \multicolumn{3}{|l|}{$\mathrm{Fe}^{2+}$} & \multirow{2}{*}{ 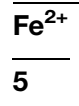 } & \multirow{2}{*}{$\frac{\mathrm{Fe}^{3+}}{6}$} \\
\hline & 1 & & & 2 & & & 3 & & & 4 & & & & \\
\hline $\mathrm{T}(\mathrm{K})$ & 200 & 77 & 4.2 & 200 & 77 & 4.2 & 200 & 77 & $4.2^{1}$ & 200 & 77 & $4.2^{1}$ & n.d. ${ }^{2}$ & n.d..$^{2}$ \\
\hline$\delta\left(\mathrm{mm} \mathrm{s}^{-1}\right)$ & 0.36 & 0.41 & 0.41 & 0.36 & 0.42 & 0.42 & 0.40 & 0.43 & 0.44 & 0.54 & 0.59 & 0.61 & 0.24 & 0.26 \\
\hline$\Delta \boldsymbol{E}_{\mathbf{Q}}\left(\mathrm{mm} \mathrm{s}^{-1}\right)$ & 0.52 & 0.70 & 0.76 & 0.81 & 1.08 & 1.25 & 1.05 & 1.10 & 0.76 & 1.60 & 1.88 & 1.25 & 1.62 & 0.81 \\
\hline $\boldsymbol{\Gamma}\left(\mathrm{mm} \mathrm{s}^{-1}\right)$ & 0.32 & 0.36 & 0.50 & 0.34 & 0.36 & 0.50 & 0.38 & 0.46 & 0.46 & 0.40 & 0.46 & 0.37 & 0.35 & 0.61 \\
\hline
\end{tabular}

${ }^{1}$ The spectral components of the $[4 \mathrm{Fe}-4 \mathrm{~S}]^{1+}$ cluster at $4.2 \mathrm{~K}$ were simulated using an effective spin $\mathrm{S}=1 / 2$ and the spin-Hamiltonian formalism in the slow relaxation limit. The hyperfine coupling tensor $\boldsymbol{A} / \mu_{\mathrm{N}} \mathrm{gN}_{\mathrm{N}}$ at $4.2 \mathrm{~K}$ for subspectrum 3 has been taken as $-230,-245$, and $-220 \mathrm{kG}$ and for subspectrum 4 a 210, 110 , and $85 \mathrm{kG}$. For the field spectra the simulations representing the $[\mathrm{Fe}](\mathrm{CN})_{2} \mathrm{CO}$ moiety and the $[4 \mathrm{Fe}-4 \mathrm{~S}]^{2+}$ cluster were obtained assuming a diamagnetic state;

${ }^{2} \mathrm{~A}$ temperature dependence for the mononuclear iron site could not be detected. The $\mathrm{Fe}^{3+}$ component was simulated with a zero field splitting $\mathrm{D}=2 \mathrm{~cm}^{-1}$ and a rhombicity $E / D=0.33$ as well as $\boldsymbol{A} / \mu_{N} g_{N}=(-200,-200,-200) \mathrm{kG}$ in the slow relaxation limit for $B=5 T$ and in the fast relaxation limit at $B=0.1 \mathrm{~T}$ (Supplementary Figure S7); Mössbauer parameters of the metal centers of the as-isolated, reduced, and oxidized form of the HypCD complex as obtained from the simulations given in Figure 6 and Supplementary Figure S7, S8. $\delta$ : Isomer shift; $\Delta E_{Q}$ : Quadrupole splitting; $\Gamma$ line width at half maximum; A: Hyperfine coupling tensor. 

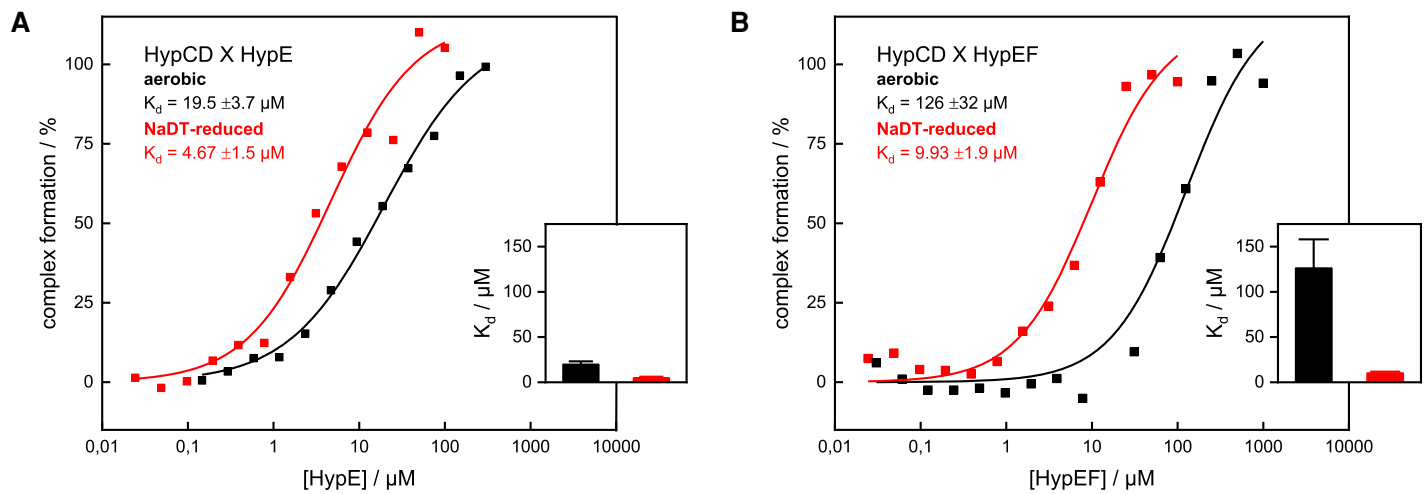

Figure 7. Redox dependency of the binding affinities between HypCD and HypE(F).

(A) HypE dependence of the MST signal for the fluorescent-labeled HypCD complex under aerobic conditions (black) or reducing conditions (1 $\mathrm{mM} \mathrm{NaDT}$, red). (B) HypEF dependence of the MST signal for the fluorescent-labeled HypCD complex under aerobic conditions (black) or reducing conditions (1 mM NaDT, red). The MST signals of at least 12 different concentrations of HypE or HypEF ranging from 0.01-1000 $\mu \mathrm{M}$ were fitted according to Michaelis-Menten kinetics. The insets in panel $(\mathbf{A})$ and $(\mathbf{B})$ depict the obtained binding affinities, $K_{\mathrm{d}}$. Data of at least three independent measurements $(\mathrm{n})$ were combined to create one dataset for which the averaged data points as the mean \pm SD (standard deviation) were fitted by Nano Temper analysis software using a $K_{\mathrm{d}}$-model. Our data show significantly higher binding affinities of HypCD to HypE and HypEF under reducing conditions.

conditions $\left(K_{\mathrm{d}}=19.5 \pm 3.7 \mu \mathrm{M}\right)$. Similar results were obtained for the interaction between HypCD and the HypEF complex (Figure 7B), indicating preferable binding under reducing conditions with $K_{\mathrm{d}}=9.93 \pm 1.9 \mu \mathrm{M}$ compared with aerobic conditions $\left(K_{\mathrm{d}}=126 \pm 32 \mu \mathrm{M}\right)$. Based on the crystal structure of the HypCDE [50] and HypEF complexes [51], it seems that interface between HypE and HypF overlaps with that between HypE and HypCD, which may explain the approximately six-fold higher binding affinity of HypE to HypCD in the absent of HypF under aerobic conditions. Conceivably, the regulation of the maturation process is based on specific molecular interactions between individual subunits. The difference in $K_{\mathrm{d}}$ is less pronounced under reducing conditions, but the trend is the same. Although the exact nature of the interactions and the temporal sequence of events has not been resolved in detail, the binding and release of HypE from the HypCD complex must be initiated by an interaction with other maturation protein(s) or by the delivery of the $[\mathrm{Fe}](\mathrm{CN})_{2} \mathrm{CO}$ cofactor precursor to the pro-protein.

\section{Discussion}

The biosynthesis of the $[\mathrm{Fe}](\mathrm{CN})_{2} \mathrm{CO}$ cofactor precursor is a complex and dynamic process catalyzed by the coordinated activity of HypCDEF $[12,13]$. While HypE and HypF catalyze the synthesis of the $\mathrm{CN}^{-}$ligand through carbamoylation and cyanation of the C-terminal cysteine of HypE [22], the maturation proteins HypC and HypD form the HypCD scaffold complex, where the $\mathrm{CN}^{-}$and $\mathrm{CO}$ ligands are attached to the iron ion to form the $[\mathrm{Fe}](\mathrm{CN})_{2} \mathrm{CO}$ cofactor precursor [12,27]. It has been hypothesized that the input of two electrons is a prerequisite for the reductive transfer of each $\mathrm{CN}^{-}$ligand from HypE to the HypCD complex [26,28,52], and minimally two further electrons would be required for the generation of $\mathrm{CO}$ if the substrate is $\mathrm{CO}_{2}[20]$. Among the four maturation proteins only HypD contains an iron-sulfur cluster and is therefore the only Hyp protein capable of performing redox chemistry; however, not much is known about the mechanism by which the $\mathrm{CN}^{-}$and $\mathrm{CO}$ ligands are transferred and attached to the iron atom on HypCD. Therefore, an efficient expression and purification system was developed to produce the HypCD complex with complete cluster and cofactor content. The successful enrichment with ${ }^{57} \mathrm{Fe}$ suggests that the approaches developed herein can be generally applied for other metalloproteins.

The crystal structure of HypD from Thermococcus kodakaraensis (TkHypD) revealed the existence of a redox cascade composed of an [4Fe-4S] cluster and two pairs of disulfide motifs SS1 and SS2 [50,53]. According to the computational model from Albareda and colleagues, the cascade also involves the $[\mathrm{Fe}]\left(\mathrm{CN}_{2}\right) \mathrm{CO}$ moiety at the interface of HypD and HypC [54]. Although the disulfide bond SS1 is not conserved in HypD from E. coli [55], the distance between the [4Fe-4S] cluster and the conserved disulfide motif SS2 is short enough to 

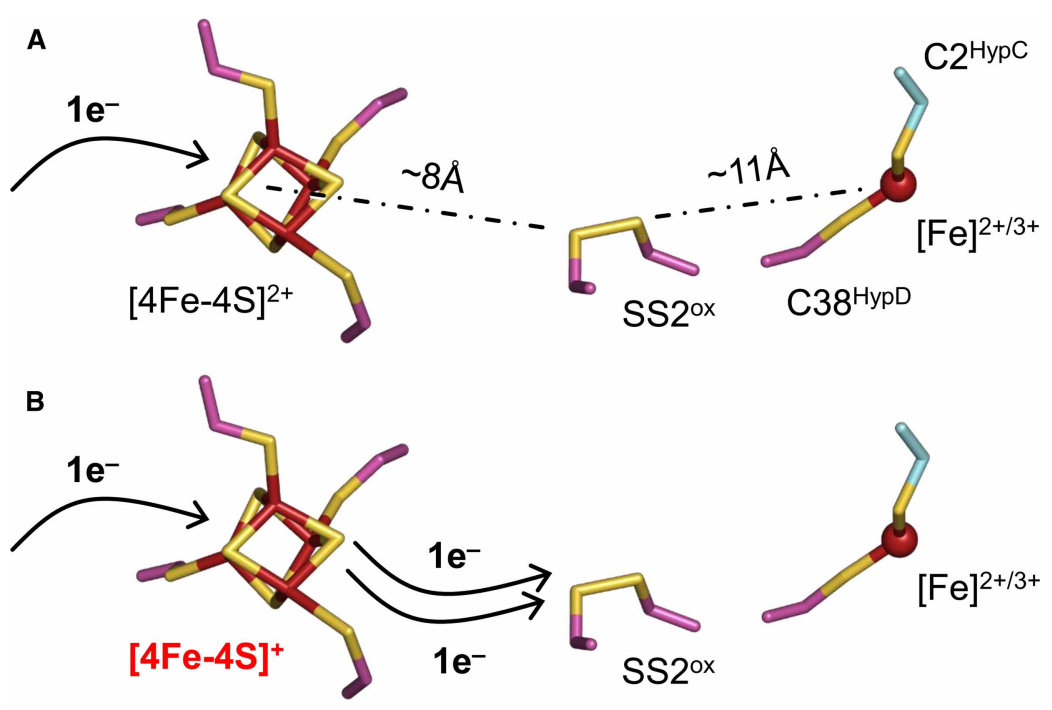

C


Figure 8. Proposed mechanism for the assembly of the $[\mathrm{Fe}](\mathrm{CN})_{2} \mathrm{CO}$ cofactor precursor.

(A) The distance between [4Fe-4S] cluster, disulfide motif SS2, and mononuclear iron site allows for efficient electron transfer. The disulfide bond $\left(\mathrm{SS} 2^{\mathrm{ox}}\right.$ ) correlates with an oxidized iron site, $[\mathrm{Fe}]^{3+}$. (B) Reduction in the $[4 \mathrm{Fe}-4 \mathrm{~S}]^{2+}$ cluster by an external electron donor to form [4Fe-4S $]^{+}$for the reduction in disulfide bridge SS2 ${ }^{\mathrm{ox}}$ to SS2 ${ }^{\text {red }}$ (red) in two consecutive one-electron transfer steps. (C) Modification of the iron site with one $\mathrm{CO}$ and two $\mathrm{CN}^{-}$ligands at the expense of $\mathrm{SS}^{\text {red }}$ to form the $[\mathrm{Fe}]^{2}$ ${ }^{+}(\mathrm{CN})_{2} \mathrm{CO}$ cofactor precursor. Per ligand, two electrons are consumed but the exact number of electrons is unclear.

facilitate electron transfer along the redox cascade (Figure 8A), e.g. facilitating reductive ligand transfer to the iron site [54]. This redox cascade has been proposed to provide reducing equivalents for the synthesis and attachment of ligands to the cofactor precursor, in agreement with our infrared data and the faradaic currents measured upon oxidation and reduction in disulfide motif SS2. Formation and excision of SS2 on the HypCD complex from E. coli is indicative of a two-electron reaction that comprises two consecutive one-electron transfer steps [56,57]. Our data now confirm the redox activity of the [4Fe-4S] cluster and suggest an electron confurcation mechanism that involves two one-electron transfer steps to form the reduced disulfide motif $\mathrm{SS}^{\text {red }}$ (Figure 8B). As discussed earlier [50,52,53], oxidation of SS2 ${ }^{\text {red }}$ would supply two electrons for the synthesis of each diatomic ligand (Figure 8C).

Previous studies demonstrated that the $\mathrm{CN}^{-}$transfer did not occur when the HypCD complex was treated with oxidizing agents or was purified under aerobic conditions, in which the disulfide bond was formed $[26,28,52]$. The inhibition of $\mathrm{CN}^{-}$transfer under oxidizing conditions seems to be due to the formation of disulfide bond $\mathrm{SS}^{\mathrm{ox}}$, which would interrupt the redox cascade (Figure 8). This result is in agreement with our biomolecular interaction studies that indicate up to 12-fold higher binding affinity between HypCD and HypEF under reducing conditions. The preferable binding under reducing conditions is likely to facilitate electron transfer during [Fe] $(\mathrm{CN})_{2} \mathrm{CO}$ cofactor synthesis. Furthermore, in the absence of reductant or under aerobic conditions, the cofactor of [NiFe]-hydrogenase cannot be assembled in vitro [12,19,58]. We conclude that $\mathrm{NaDT}$ serves as sacrificial reductant in the in vitro assay, replacing the role of the physiological electron donor of HypD.

The finding that $\mathrm{HypC}$ coordinates an iron-bound $\mathrm{CO}_{2}$ molecule suggests that the HypC superfamily may deliver both iron and $\mathrm{CO}_{2}$ for cofactor biosynthesis [20]. Our working hypothesis comprises reduction in $\mathrm{CO}_{2}$ 
with $2 \mathrm{e}^{-}$and $2 \mathrm{H}^{+}$to $\mathrm{CO}$ and $\mathrm{H}_{2} \mathrm{O}$ as catalyzed by the HypCD complex; however, the proposed reaction requires a low redox potential $\left(\mathrm{E}_{0}{ }^{\prime} \approx-530 \mathrm{mV}\right)$. It has been demonstrated that the HypCD complex catalyzes the hydrolysis of ATP [21], which would help to overcome such thermodynamic barriers, not unlike CO dehydrogenase [59], the 'iron protein' of nitrogenase [60], or enoyl-CoA reductase [61]. Under aerobic conditions, the CO ligand is generated by decarbonylating formyltetrahydrofolate $[62,63]$. However, the metabolic source in strict or facultative anaerobes must be different. To this end, our spectroscopic evaluation of the HypCD scaffold complex helps to define the different redox states of the $[4 \mathrm{Fe}-4 \mathrm{~S}]$ cluster and the $[\mathrm{Fe}](\mathrm{CN})_{2} \mathrm{CO}$ cofactor precursor. The data demonstrate that the $[4 \mathrm{Fe}-4 \mathrm{~S}]$ cluster is redox active and provides comprehensive evidence for an electron inventory fit to drive multi-electron redox reactions required for the assembly of the $\mathrm{CN}^{-}$and $\mathrm{CO}$ ligands on the HypCD complex.

\section{Data Availability}

Strains, mutants, and plasmids that arise from this work will be available on request. All of the primary data that is presented in this study can be requested in electronic form by contacting Basem Soboh (basem.

soboh@fu-berlin.de).

\section{Competing Interests}

The authors declare that there are no competing interests associated with the manuscript.

\section{Funding}

This work was supported by German Research Foundation (DFG) via the SPP 1927 priority program 'Iron-Sulfur for Life,' Grant No. SO1325/5-2 to B.S, STR1554/5-1 to S.T.S., AD178/7-1 to LA, SCHU1251/17-2 to V.S., and PI610/2-1 to A.J.P. Part of the project is funded by the DFG under Germany's Excellence Strategy - EXC 2008/ 1-390540038 (UniSysCat) to J.H. and R.S.

\section{CRediT Author Contribution}

Basem Soboh: Conceptualization, Resources, Data curation, Software, Formal analysis, Supervision, Funding acquisition, Validation, Investigation, Visualization, Methodology, Writing - original draft, Project administration, Writing - review and editing. Sven T. Stripp: Conceptualization, Resources, Data curation, Software, Formal analysis, Supervision, Funding acquisition, Validation, Investigation, Visualization, Methodology, Writing original draft, Project administration, Writing - review and editing. Jonathan Oltmanns: Formal analysis, Investigation, Methodology, Writing - original draft. Christina S. Müller: Methodology. David Ehrenberg: Methodology. Ramona Schlesinger: Project administration. Joachim Heberle: Funding acquisition, Methodology. Lorenz Adrian: Formal analysis, Supervision, Funding acquisition, Methodology. Volker Schünemann: Formal analysis, Supervision, Funding acquisition, Validation, Methodology, Writing - original draft. Antonio J. Pierik: Formal analysis, Supervision, Funding acquisition, Validation, Methodology.

\section{Acknowledgements}

We gratefully acknowledge financial support from German Research Foundation (DFG) via the SPP 1927 priority program 'Iron-Sulfur for Life'. Protein mass spectrometry was done at the Centre for Chemical Microscopy (ProVIS) at UFZ Leipzig.

\section{Abbreviations}

ATR, Attenuated total reflection; EPR, electron paramagnetic resonance; FTIR, Fourier-transform infrared; ICP-QqQ-MS, inductively coupled plasma mass spectrometry; MST, MicroScale thermophoresis; PMS, phenazine methosulfate; RR, Resonance Raman.

\section{References}

1 Lu, Y., Yeung, N., Sieracki, N. and Marshall, N.M. (2009) Design of functional metalloproteins. Nature 460, 855-862 https://doi.org/10.1038/ nature08304

2 Thauer, R.K., Kaster, A.K., Goenrich, M., Schick, M., Hiromoto, T. and Shima, S. (2010) Hydrogenases from methanogenic archaea, nickel, a novel cofactor, and H-2 storage. Annu. Rev. Biochem. 79, 507-536 https://doi.org/10.1146/annurev.biochem.030508.152103

3 Vignais, P.M. and Billoud, B. (2007) Occurrence, classification, and biological function of hydrogenases: an overview. Chem. Rev. 107, 4206-4272 https://doi.org/10.1021/cr050196r

4 Shafaat, H.S., Rudiger, O., Ogata, H. and Lubitz, W. (2013) [Nife] hydrogenases: a common active site for hydrogen metabolism under diverse conditions. Biochim. Biophys. Acta 1827, 986-1002 https://doi.org/10.1016/j.bbabio.2013.01.015 
5 Lubitz, W., Ogata, H., Rudiger, O. and Reijerse, E. (2014) Hydrogenases. Chem. Rev. 114, 4081-4148 https://doi.org/10.1021/cr4005814

6 Cammack, R., Frey, M. and Robson, R. (2001) Hydrogen as a fuel Learning from Nature

7 Shomura, Y., Taketa, M., Nakashima, H., Tai, H., Nakagawa, H., Ikeda, Y. et al. (2017) Structural basis of the redox switches in the NAD(+)-reducing soluble [NiFe]-hydrogenase. Science 357, 928-932 https://doi.org/10.1126/science.aan4497

8 McTernan, P.M., Chandrayan, S.K., Wu, C.H., Vaccaro, B.J., Lancaster, W.A., Yang, Q.Y. et al. (2014) Intact functional fourteen-subunit respiratory membrane-bound [NiFe]-hydrogenase complex of the hyperthermophilic archaeon Pyrococcus furiosus. J. Biol. Chem. 289, 19364-19372 https://doi. org/10.1074/jbc.M114.567255

9 Burgdorf, T., Lenz, O., Buhrke, T., van der Linden, E., Jones, A.K., Albracht, S.P.J. et al. (2005) [Nife]-hydrogenases of Ralstonia eutropha H16: Modular enzymes for oxygen-tolerant biological hydrogen oxidation. J. Mol. Microbiol. Biotechnol. 10, 181-196 https://doi.org/10.1159/000091564

10 Happe, R.P., Roseboom, W., Pierik, A.J., Albracht, S.P. and Bagley, K.A. (1997) Biological activation of hydrogen. Nature 385, 126 https://doi.org/10 $1038 / 385126 a 0$

11 Pierik, A.J., Roseboom, W., Happe, R.P., Bagley, K.A. and Albracht, S.P. (1999) Carbon monoxide and cyanide as intrinsic ligands to iron in the active site of [NiFe]-hydrogenases. NiFe(CN) $)_{2} \mathrm{CO}$, Biology's way to activate H2. J. Biol. Chem. 274, 3331-3337 https://doi.org/10.1074/jbc.274.6.3331

12 Senger, M., Stripp, S.T. and Soboh, B. (2017) Proteolytic cleavage orchestrates cofactor insertion and protein assembly in [NiFe]-hydrogenase biosynthesis. J Biol Chem 292, 11670-11681 https://doi.org/10.1074/jbc.M117.788125

13 Böck, A., King, P.W., Blokesch, M. and Posewitz, M.C. (2006) Maturation of hydrogenases. Adv Microb Physio/ 51, 1-71 https://doi.org/10.1016/ S0065-2911(06)51001-X

14 Lacasse, M.J., Douglas, C.D. and Zamble, D.B. (2016) Mechanism of selective nickel transfer from HypB to HypA, Escherichia coli [NiFe]-hydrogenase accessory proteins. Biochemistry 55, 6821-6831 https://doi.org/10.1021/acs.biochem.6b00706

15 Soboh, B., Kuhns, M., Braussemann, M., Waclawek, M., Muhr, E., Pierik, A.J. et al. (2012) Evidence for an oxygen-sensitive iron-sulfur cluster in an immature large subunit species of Escherichia coli [NiFe]-hydrogenase 2. Biochem. Biophys. Res. Commun. 424, 158-163 https://doi.org/10.1016/j. bbrc.2012.06.096

16 Watanabe, S., Kawashima, T., Nishitani, Y., Kanai, T., Wada, T., Inaba, K. et al. (2015) Structural basis of a Ni acquisition cycle for [NiFe] hydrogenase by Ni-metallochaperone HypA and its enhancer. Proc. Natl Acad. Sci. U.S.A. 112, 7701-7706 https://doi.org/10.1073/pnas.1503102112

17 Pinske, C., Kruger, S., Soboh, B., Ihling, C., Kuhns, M., Braussemann, M. et al. (2011) Efficient electron transfer from hydrogen to benzyl viologen by the [NiFe]-hydrogenases of Escherichia coli is dependent on the coexpression of the iron-sulfur cluster-containing small subunit. Arch. Microbiol. 193, 893-903 https://doi.org/10.1007/s00203-011-0726-5

18 Thomas, C., Muhr, E. and Sawers, R.G. (2015) Coordination of synthesis and assembly of a modular membrane-associated [NiFe]-hydrogenase is determined by cleavage of the C-terminal peptide. J. Bacteriol. 197, 2989-2998 https://doi.org/10.1128/JB.00437-15

19 Soboh, B., Lindenstrauss, U., Granich, C., Javed, M., Herzberg, M., Thomas, C. et al. (2014) [Nife]-hydrogenase maturation in vitro: analysis of the roles of the HybG and HypD accessory proteins. Biochem. J. 464, 169-177 https://doi.org/10.1042/BJ20140485

20 Soboh, B., Stripp, S.T., Bielak, C., Lindenstrauss, U., Braussemann, M., Javaid, M. et al. (2013) The [NiFe]-hydrogenase accessory chaperones HypC and HybG of Escherichia coli are iron- and carbon dioxide-binding proteins. FEBS Lett. 587, 2512-2516 https://doi.org/10.1016/j.febslet.2013.06.055

21 Nutschan, K., Golbik, R.P. and Sawers, R.G. (2019) The iron-sulfur-containing HypC-HypD scaffold complex of the [NiFe]-hydrogenase maturation machinery is an ATPase. FEBS Open Bio 9, 2072-2079 https://doi.org/10.1002/2211-5463.12743

22 Paschos, A., Bauer, A., Zimmermann, A., Zehelein, E. and Böck, A. (2002) Hypf, a carbamoyl phosphate-converting enzyme involved in [NiFe] hydrogenase maturation. J. Biol. Chem. 277, 49945-49951 https://doi.org/10.1074/jbc.M204601200

23 Stripp, S.T., Lindenstrauss, U., Granich, C., Sawers, R.G. and Soboh, B. (2014) The influence of oxygen on [NiFe]-hydrogenase cofactor biosynthesis and how ligation of carbon monoxide precedes cyanation. PLOS ONE 9, e107488 https://doi.org/10.1371/journal.pone.0107488

24 Soboh, B., Stripp, S.T., Muhr, E., Granich, C., Braussemann, M., Herzberg, M. et al. (2012) [Nife]-hydrogenase maturation: isolation of a HypC-HypD complex carrying diatomic CO and CN- ligands. FEBS Lett. 586, 3882-3887 https://doi.org/10.1016/j.febslet.2012.09.019

25 Stripp, S.T., Soboh, B., Lindenstrauss, U., Braussemann, M., Herzberg, M., Nies, D.H. et al. (2013) Hypd is the scaffold protein for Fe-(CN)2CO cofactor assembly in [NiFe]-hydrogenase maturation. Biochemistry 52, 3289-3296 https://doi.org/10.1021/bi400302v

26 Blokesch, M., Albracht, S.P., Matzanke, B.F., Drapal, N.M., Jacobi, A. and Böck, A. (2004) The complex between hydrogenase-maturation proteins HypC and HypD is an intermediate in the supply of cyanide to the active site iron of [NiFe]-hydrogenases. J. Mol. Biol. 344, 155-167 https://doi.org/10.1016/ j.jmb.2004.09.04a

27 Burstel, I., Siebert, E., Winter, G., Hummel, P., Zebger, I., Friedrich, B. et al. (2012) A universal scaffold for synthesis of the Fe(CN)2(CO) moiety of [NiFe] hydrogenase. J. Biol. Chem. 287, 38845-38853 https://doi.org/10.1074/jbc.M112.376947

28 Roseboom, W., Blokesch, M., Bock, A. and Albracht, S.P. (2005) The biosynthetic routes for carbon monoxide and cyanide in the Ni-Fe active site of hydrogenases are different. FEBS Lett. 579, 469-472 https://doi.org/10.1016/j.febslet.2004.12.013

29 Seidel, K., Kuhnert, J. and Adrian, L. (2018) The complexome of Dehalococcoides mccartyi reveals its organohalide respiration-complex is modular Front. Microbiol. 9, 1130 https://doi.org/10.3389/fmicb.2018.01130

30 Jacobi, A., Rossmann, R. and Bock, A. (1992) The hyp operon gene products are required for the maturation of catalytically active hydrogenase isoenzymes in Escherichia coli. Arch. Microbiol. 158, 444-451 https://doi.org/10.1007/BF00276307

31 Ballantine, S.P. and Boxer, D.H. (1985) Nickel-containing hydrogenase isoenzymes from anaerobically grown Escherichia coli K-12. J. Bacteriol. 163, 454-459 https://doi.org/10.1128/jb.163.2.454-459.1985

32 Lowry, O.H., Rosebrough, N.J., Farr, A.L. and Randall, R.J. (1951) Protein measurement with the Folin phenol reagent. J. Biol. Chem. 193, 265-275 https://doi.org/10.1016/S0021-9258(19)52451-6

33 Budhraja, R., Ding, C., Walter, P., Wagner, S., Reemtsma, T., Gary Sawers, R. et al. (2019) The impact of species, respiration type, growth phase and genetic inventory on absolute metal content of intact bacterial cells. Metallomics 11, 925-935 https://doi.org/10.1039/C9MT00009G

34 Radu, I., Bamann, C., Nack, M., Nagel, G., Bamberg, E. and Heberle, J. (2009) Conformational changes of channelrhodopsin-2. J. Am. Chem. Soc. 131, 7313-7319 https://doi.org/10.1021/ja8084274

35 Stripp, S.T. (2021) In situ infrared spectroscopy for the analysis of gas-processing metalloenzymes. Acs Catalysis 11, 7845-7862 https://doi.org/10. 1021/acscatal.1c00218 
36 Senger, M., Mebs, S., Duan, J.F., Shulenina, O., Laun, K., Kertess, L. et al. (2018) Protonation/reduction dynamics at the [4Fe-4S] cluster of the hydrogen-forming cofactor in [FeFe]-hydrogenases. Phys. Chem. Chem. Phys. 20, 3128-3140 https://doi.org/10.1039/C7CP04757F

37 Jerabek-Willemsen, M., Andre, T., Wanner, R., Roth, H.M., Duhr, S., Baaske, P. et al. (2014) Microscale thermophoresis: interaction analysis and beyond. J. Mol. Struct. 1077, 101-113 https://doi.org/10.1016/j.molstruc.2014.03.009

38 Yeliseev, A., Zoubak, L. and Schmidt, T.G.M. (2017) Application of Strep-Tactin XT for affinity purification of Twin-Strep-tagged CB2, a G protein-coupled cannabinoid receptor. Protein Express Purif. 131, 109-118 https://doi.org/10.1016/j.pep.2016.11.006

39 Sweeney, W.V. and Rabinowitz, J.C. (1980) Proteins containing 4fe-4s clusters: an overview. Annu. Rev. Biochem. 49, 139-161 https://doi.org/10. 1146/annurev.bi.49.070180.001035

40 Czernuszewicz, R.S., Macor, K.A., Johnson, M.K., Gewirth, A. and Spiro, T.G. (1987) Vibrational-mode structure and symmetry in proteins and analogs containing Fe4s4 clusters: resonance Raman evidence for different degrees of distortion in hipip and ferredoxin. J. Am. Chem. Soc. 109, 7178-7187 https://doi.org/10.1021/ja00257a045

41 Barth, A. (2007) Infrared spectroscopy of proteins. Biochim. Biophys. Acta 1767, 1073-1101 https://doi.org/10.1016/j.bbabio.2007.06.004

42 Mayhew, S.G. (1978) The redox potential of dithionite and S0-2 from equilibrium reactions with flavodoxins, methyl viologen and hydrogen plus hydrogenase. Eur. J. Biochem. 85, 535-547 https://doi.org/10.1111/j.1432-1033.1978.tb12269.x

43 Cammack, R. (1988) Electron-paramagnetic resonance characterization of iron sulfur proteins. Methods Enzymol. 167, 427-436 https://doi.org/10. 1016/0076-6879(88)67047-9

44 White, J.R. and Dearman, H.H. (1965) Generation of free radicals from phenazine methosulfate, streptonigrin, and riboflavin in bacterial suspensions. Proc. Natl Acad. Sci. U.S.A. 54, 887-891 https://doi.org/10.1073/pnas.54.3.887

45 Priem, A.H., Klaassen, A.A., Reijerse, E.J., Meyer, T.E., Luchinat, C., Capozzi, F. et al. (2005) EPR analysis of multiple forms of [4Fe-4S](3+) clusters in HiPIPs. J. Biol. Inorg. Chem. 10, 417-424 https://doi.org/10.1007/s00775-005-0656-2

46 Pandelia, M.E., Lanz, N.D., Booker, S.J. and Krebs, C. (2015) Mossbauer spectroscopy of Fe/S proteins. Biochim. Biophys. Acta 1853, 1395-1405 https://doi.org/10.1016/.jbbamcr.2014.12.005

47 Middleton, P., Dickson, D.P., Johnson, C.E. and Rush, J.D. (1978) Interpretation of the Mossbauer spectra of the four-iron ferredoxin from Bacillus stearothermophilus. Eur. J. Biochem. 88, 135-141 https://doi.org/10.1111/j.1432-1033.1978.tb12430.x

48 Kyritsis, P., Kummerle, R., Huber, J.G., Gaillard, J., Guigliarelli, B., Popescu, C. et al. (1999) Unusual NMR, EPR, and Mossbauer properties of Chromatium vinosum 2[4Fe-4S] ferredoxin. Biochemistry 38, 6335-6345 https://doi.org/10.1021/bi982894u

49 Roncaroli, F., Bill, E., Friedrich, B., Lenz, O., Lubitz, W. and Pandelia, M.E. (2015) Cofactor composition and function of a H2-sensing regulatory hydrogenase as revealed by Mossbauer and EPR spectroscopy. Chem. Sci. 6, 4495-4507 https://doi.org/10.1039/C5SC01560J

50 Watanabe, S., Matsumi, R., Atomi, H., Imanaka, T. and Miki, K. (2012) Crystal structures of the HypCD complex and the HypCDE ternary complex: transient intermediate complexes during [NiFe] hydrogenase maturation. Structure 20, 2124-2137 https://doi.org/10.1016/j.str.2012.09.018

51 Shomura, Y. and Higuchi, Y. (2012) Structural basis for the reaction mechanism of S-carbamoylation of HypE by HypF in the maturation of [NiFe]-hydrogenases. J. Biol. Chem. 287, 28409-28419 https://doi.org/10.1074/jbc.M112.387134

52 Blokesch, M. and Bock, A. (2006) Properties of the [NiFe]-hydrogenase maturation protein HypD. FEBS Lett. 580, 4065-4068 https://doi.org/10.1016/j. febslet.2006.06.045

53 Watanabe, S., Matsumi, R., Arai, T., Atomi, H., Imanaka, T. and Miki, K. (2007) Crystal structures of [NiFe] hydrogenase maturation proteins HypC, HypD, and HypE: Insights into cyanation reaction by thiol redox signaling. Mol. Cell 27, 29-40 https://doi.org/10.1016/j.molcel.2007.05.039

54 Albareda, M., Palacios, J.M., Imperial, J. and Pacios, L.F. (2013) Computational study of the Fe(CN)(2)CO cofactor and its binding to HypC protein. J. Phys. Chem. B 117, 13523-13533 https://doi.org/10.1021/jp407574n

55 Watanabe, S., Matsumi, R., Atomi, H., Imanaka, T. and Miki, K. (2007) Crystallization and preliminary X-ray crystallographic studies of the [NiFe] hydrogenase maturation proteins HypC and HypD. Acta Crystallogr. Sect. F Struct. Biol. Cryst. Commun. 63, 538-541 https://doi.org/10.1107/ S1744309107023391

56 Adamson, H., Robinson, M., Bond, P.S., Soboh, B., Gillow, K., Simonov, A.N. et al. (2017) Analysis of HypD disulfide redox chemistry via optimization of Fourier transformed ac voltammetric data. Anal. Chem 89, 1565-1573 https://doi.org/10.1021/acs.analchem.6b03589

57 Dale-Evans, A.R., Robinson, M.J., Lloyd-Laney, H.O., Gavaghan, D.J., Bond, A.M. and Parkin, A. (2021) A voltammetric perspective of multi-electron and proton transfer in protein redox chemistry: insights from computational analysis of Escherichia coli HypD Fourier transformed alternating current voltammetry. Front. Chem. 9, 672831 https://doi.org/10.3389/fchem.2021.672831

58 Soboh, B., Kruger, S., Kuhns, M., Pinske, C., Lehmann, A. and Sawers, R.G. (2010) Development of a cell-free system reveals an oxygen-labile step in the maturation of [NiFe]-hydrogenase 2 of Escherichia coli. FEBS Lett. 584, 4109-4114 https://doi.org/10.1016/j.febslet.2010.08.037

59 Jeoung, J.H. and Dobbek, H. (2007) Carbon dioxide activation at the Ni,Fe-cluster of anaerobic carbon monoxide dehydrogenase. Science $\mathbf{3 1 8}$, 1461-1464 https://doi.org/10.1126/science.1148481

60 Rebelein, J.G., Stiebritz, M.T., Lee, C.C. and Hu, Y.L. (2017) Activation and reduction of carbon dioxide by nitrogenase iron proteins. Nat. Chem. Biol. 13, 147-149 https://doi.org/10.1038/nchembio.2245

61 Stoffel, G.M.M., Saez, D.A., DeMirci, H., Vogeli, B., Rao, Y., Zarzycki, J. et al. (2019) Four amino acids define the C02 binding pocket of enoyl-CoA carboxylases/reductases. Proc. Natl Acad. Sci. U.S.A. 116, 13964-13969 https://doi.org/10.1073/pnas.1901471116

62 Burstel, I., Siebert, E., Frielingsdorf, S., Zebger, I., Friedrich, B. and Lenz, O. (2016) CO synthesized from the central one-carbon pool as source for the iron carbonyl in $\mathrm{O}_{2}$-tolerant [NiFe]-hydrogenase. Proc. Natl Acad. Sci. U.S.A. 113, 14722-14726 https://doi.org/10.1073/pnas.1614656113

63 Schulz, A.C., Frielingsdorf, S., Pommerening, P., Lauterbach, L., Bistoni, G., Neese, F. et al. (2020) Formyltetrahydrofolate decarbonylase synthesizes the active site $\mathrm{CO}$ ligand of $\mathrm{O}_{2}$-tolerant [NiFe] hydrogenase. J. Am. Chem. Soc. 142, 1457-1464 https://doi.org/10.1021/jacs.9b11506 